\title{
FAR-INFRARED SPECTRAL OBSERVATIONS OF THE GALAXY BY THE FAR-INFRARED ABSOLUTE SPECTROPHOTOMETER
}

\author{
WILLIAM T. REACH \\ Universities Space Research Corporation \\ NASA/GSFC, Code 685, Greenbelt, MD 20771
}

\begin{abstract}
The Galactic continuum spectrum from $5-96 \mathrm{~cm}^{-1}$ is derived from COBE $^{1}$ /FIRAS observations. The spectra are dominated by warm dust emission, which may be fit with a single temperature along each line of sight in the range 16-21 K. A widespread, very cold component $(4-7 \mathrm{~K})$ with optical depth that is spatially correlated with the warm component is also detected. The nature of the cold component and its implications for the amount of very cold material in the Milky Way are discussed.
\end{abstract}

\section{Introduction}

The all-sky observations by the Far Infrared Absolute Spectrophotometer (FIRAS) aboard the Cosmic Background Explorer (COBE), provide the first complete coverage of the wavelength range from $4.5 \mathrm{~mm}$ through $104 \mu \mathrm{m}$. Here we present observations of the galactic continuum spectrum and its variation both in the galactic plane and at high galactic latitude. Full details of this, work are presented in [6].

\section{Observations and Method}

The FIRAS has a $7^{\circ}$ beam and it detects frequencies $2.2-96 \mathrm{~cm}^{-1}$ (wavelengths $4.5 \mathrm{~mm}-104 \mu \mathrm{m}$ ), with a spectral resolution of $0.57 \mathrm{~cm}^{-1}$ over the

\footnotetext{
${ }^{1}$ The National Aeronautics and Space Administration/ Goddard Space Flight Center (NASA/GSFC) is responsible for the design, development, and operation of the Cosmic Background Explorer $(C O B E)$. Scientific guidance is provided by the $C O B E$ Science Working Group. FSDC is also responsible for the development of the analysis software and for the production of the mission data sets.
} 
entire spectral range; see [3] for details. The spectra are dominated by the cosmic background radiation, interstellar medium, and zodiacal light (at high frequencies). The cosmic microwave background radiation was removed from each spectrum by subtracting a blackbody. The zodiacal light was modeled using Diffuse Infrared Background Experiment observations, to which a parameterized model of the density and temperature of interplanetary dust was fitted. Spectral lines were avoided by eliminating the appropriate frequencies.

Coaddition was necessary in order to reduce the noise in the spectra at high galactic latitude. The spectra were combined into 120 longitude bins in the galactic plane and 26 high-latitude regions. Two sample spectra, toward $\left(l=45^{\circ}, b=0^{\circ}\right)$ and $\left(90^{\circ}>l>0^{\circ},-30^{\circ}>b>-60^{\circ}\right)$ are shown in Figures 1(a) and 2(a).

The spectrum for each region was fitted by one or more modified blackbodies, with a power-law emissivity, $\epsilon_{\nu}=\left(\nu / \nu_{0}\right)^{\alpha}$. In the low-frequency limit, the Kramers-Kronig theorem can be used to show that $\alpha$ is an even positive integer [9], although it is not yet known below which frequency this limit applies. The results of using a $\nu^{2}$ emissivity, a $\nu^{\alpha}$ emissivity, and a $\nu^{2}$ emissivity with two temperatures are shown for the two sample spectra in Figures $1(b-d)$ and $2(b-d)$.

\section{Results}

In the galactic plane, the FIRAS spectra cannot be modeled by a single modified blackbody with a power-law emissivity. Neither are the spectra well fitted by a continuous distribution of temperatures centered on the temperature of the dominant component at $16-21 \mathrm{~K}$. Rather, the observations indicate an excess that can be reproduced by a second temperature component at much lower temperature (4-7 K). Just off the galactic plane, in the $10^{\circ}<b<30^{\circ}$ zone, the very cold component is also required to match the spectra. At the highest galactic latitudes, the spectra are fit equally well by a two-temperature model or a single-temperature model with a power-law emissivity with $\alpha \simeq 1.5$. Because it is unlikely that the grain properties are significantly different at high latitude, we consider the two-component model to be valid there as well.

The optical depths of the warm and cold components are compared in Figure 3. The correlation is not exactly linear: the slope becomes steeper at fainter levels (corresponding to higher latitude). Also, the temperature of the very cold component has significant variation across the sky. In a principal components analysis of the FIRAS data, Barnes [1] identified the very cold component as an eigenspectrum with a spatial variation similar to but distinct from that of the warm dust. 


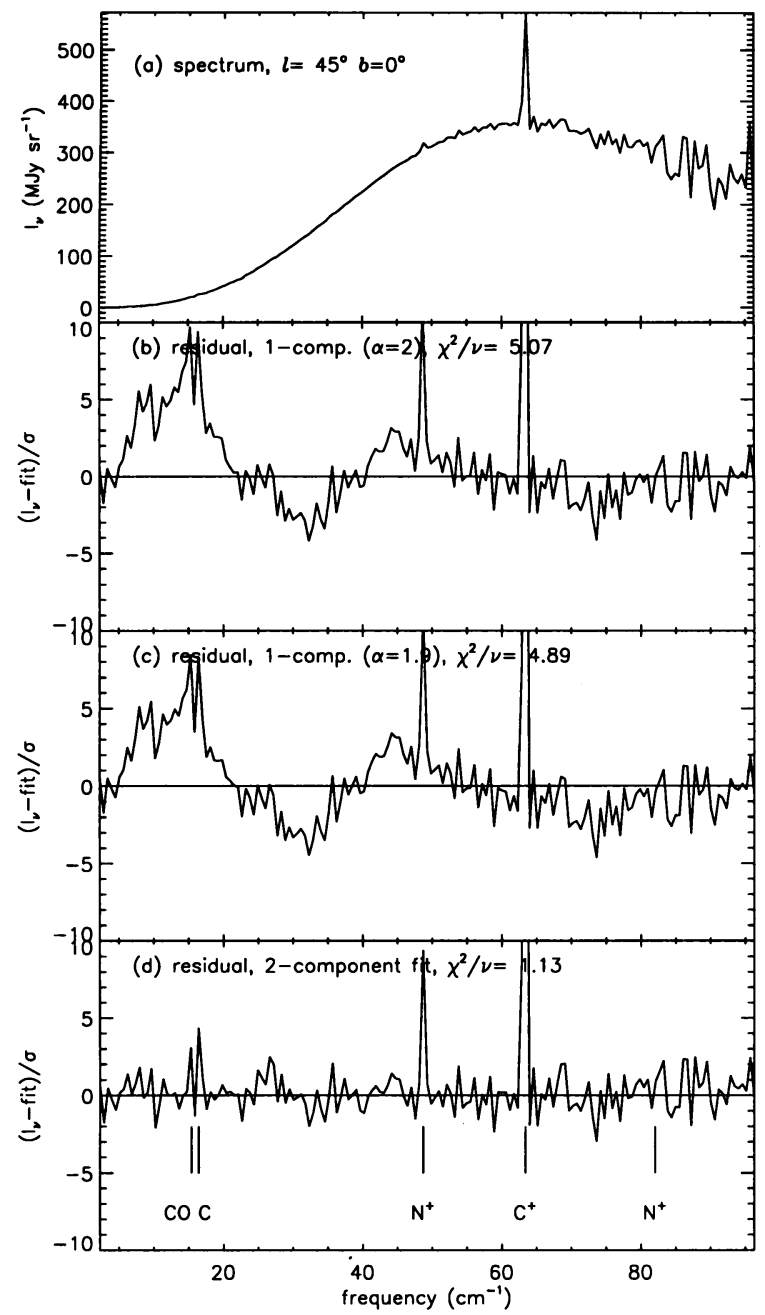

Figure 1. (a) Spectrum of interstellar emission in the galactic plane toward longitude $l=45^{\circ}$. Other than the identified spectral lines, the spectrum is dominated by emission from warm dust, which peaks around $65 \mathrm{~cm}^{-1}(150 \mu \mathrm{m})$. (b) Residuals after a single-component modified blackbody with $\alpha=2$ was subtracted. The residual intensity at each frequency was divided by the uncertainty at that frequency. The excess emission at $7-20 \mathrm{~cm}^{-1}(1400-500 \mu \mathrm{m})$ is evident. (c) Residuals after a single component with the best-fitting emissivity index was subtracted. (d) Residuals after a two-component model was subtracted. The very cold emission is now adequately fit. 


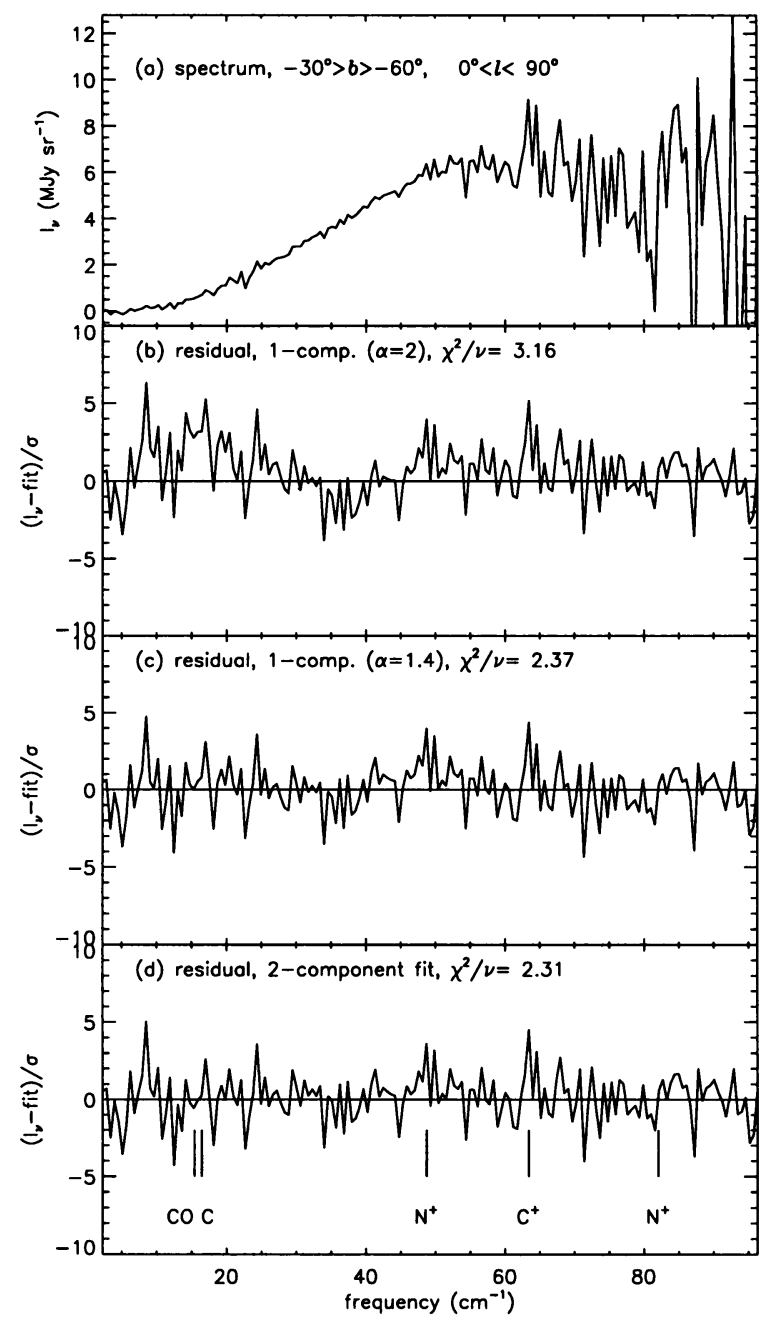

Figure 2. (a) Spectrum of interstellar emission in the region with $-30>b>-60^{\circ}$ and $90^{\circ}>l>0^{\circ}$. Only a weak $\mathrm{C}^{+}$spectral line and the warm dust continuum are evident. (b) Residuals after a single-component modified blackbody with $\alpha=2$ was subtracted. The excess emission at $7-20 \mathrm{~cm}^{-1}(1400-500 \mu \mathrm{m})$ is evident. (c) Residuals after a single component with the best-fitting emissivity index $(\alpha=1.4)$ was subtracted. (d) Residuals after a two-component model was subtracted. It is not possible to clearly distinguish whether panel (c) or (d) are better fits to the data.

\section{Nature of the Very Cold Component}

The nature of the very cold component identified in the FIRAS spectra is of great importance, because the existence of very cold material in the 


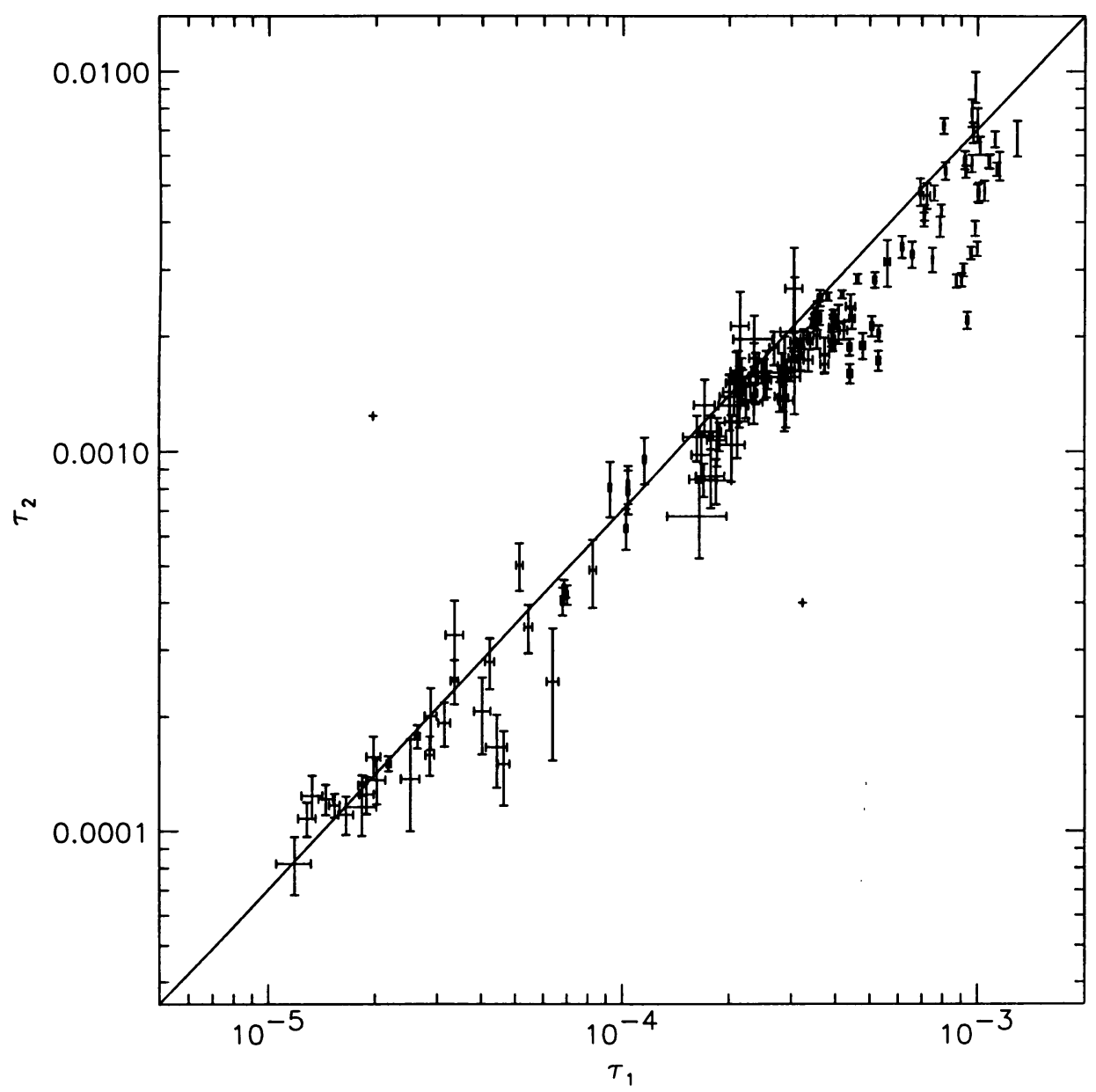

Figure 3. Comparison of the optical depths of the warm and cold components.

Milky Way has implications for the 'missing mass' problem, the composition of the halo, and the reservoir of material available for star formation. One obvious hypothesis is that the cold component is due to dust grains, similar to those that produce the warm component, but illuminated by a much-reduced radiation field. The existence of the cold component at high galactic latitude seems to preclude this hypothesis, because there are insufficient dark regions at high galactic latitude within which to shield the 
dust. The strong variation of the cold component with galactic coordinates (as reflected in Figure 3) precludes its being produced exclusively in the halo, although a small halo contribution remains possible [4].

Three more hypotheses for the nature of the very cold component are emission from large grains $\left(\sim 10^{2} \mu \mathrm{m}\right)$, very small grains $(<0.02 \mu \mathrm{m})$, or fractal grains. Large and fractal grains are cold because they emit relatively efficiently in the far-infrared and submillimeter, and very small grains are cold (most of the time) because they cool rapidly in between successive absorptions of photons. The large-grain hypothesis runs into abundance constraints on the heavy elements they contain, but at least one grain model has been able to incorporate relatively large grains without violating cosmic abundances [7]. The very small grain hypothesis requires some heating mechanism to keep the grains from cooling all the way to the cosmic background temperature; this issue has not been addressed yet. Fractal grains can attain a wide range of temperatures due to their geometry alone.

Another hypothesis for the cold component is that it is due to an emissivity enhancement in the submillimeter due to the optical properties of the constituent material. The variations in the cold component relative to the warm component would require that the composition change across the sky. This hypothesis is being explored further, in an attempt to determine the emissivity of interstellar dust by inverting the FIRAS spectra.

\section{Implications for Cold Gas in the Milky Way}

The lack of substantial power emitted by dust with temperatures in the 4-15 K range places strong constraints on the amount of very cold material in the interstellar medium. The discovery of $\mathrm{CO}$ absorption lines from clouds in the outer galaxy led to the suggestion that very cold molecular clouds, which would have been very difficult to detect by $\mathrm{CO}$ emission, may be an important, massive component of the Galaxy [5]. The power of all starlight incident on such cold clouds would be reemitted within the FIRAS bandpass. In fact, there is little power emitted besides that contained in the warm component: the cold component contains less than $0.05 \%$ of the power in the Solar neighborhood interstellar radiation field. Models for the interstellar medium including a substantial increase in the abundance of dark clouds, such as those required to harbor cold gas, must comply with the constraint that very little power is emitted by cold dust. Evidence for star formation in the far outer galaxy [2] suggests both that dust formation (in stellar outflows and supernovae) occurs there and that the radiation field will not be negligible; power absorbed from the extragalactic radiation field is also detectable. 


\section{References}

1. Barnes, W. (1994), Ph. D. thesis, M.I.T.

2. De Geus, E. J., Vogel, S. N., Digel, S. W., \& Gruendl, R. A. (1993), Astrophys. J., 413, L97.

3. Fixsen, D. J., et al. (1994), Astrophys. J., 420, 457.

4. Gerhard, O. E., \& Silk, J. (1994), preprint.

5. Lequeux, J., Allen, R. J., \& Guilloteau, S. (1993), Astron. \& Astropys., 280, L23.

6. Reach, W. T. et al.(1994), submitted to Astrophys. J..

7. Rowan-Robinson, R. (1993), Mon. Not. Roy. Astron. Soc., 258, 787.

8. Wright, E. L. (1991), Astrophys. J., 381, 200

9. Wright, E. L. (1993), in Back to the Galaxy, eds. S. S. Holt \& F. Verter (AIP: New York), p. 193. 